Ann. Biol. anim., Bioch., Biophys., I965, 5 (4), 5×3-526.

\title{
DOSAGE DE L'AMIDON DANS LES MILIEUX COMPLEXES
}

\author{
P. THIVEND, Christiane MERCIER et A. GUILBOT \\ Station de Recherches sur l'Elevage des Ruminants, \\ Centre national de Recherches zootechniques, Jouy-en-Josas (Seine-et-Oise); \\ Laboratoire de Biochimie et Physico-Chimie des Céréales au C.E.R.D.I.A., \\ Le Noyer-Lambert, Massy (Seine-et-Oise)
}

\section{SOMMAIRE}

Les méthodes de dosage de l'amidon sont nombreuses, mais aucune n'est à la fois spécifique et rapide. La recherche d'une méthode présentant simultanément ces deux qualités nous a conduits à proposer un dosage par voie enzymatique. Le système enzymatique utilisé est une gluco-amylase d'origine fongi fue qui est susceptible de transformer quantitativement l'amidon en glucose, par rupture des liaisons $\alpha(\mathrm{I} \rightarrow 4)$ et $\alpha(\mathrm{I} \rightarrow 6)$. Le glucose de l'hydrolysat est déterminé par la méthode colorimétrique de Huggett (1957) modifiée.

L'étude des modalités d'hydrolyse nous a permis de préciser les conditions optimales d'accessibilité du substrat à l'enzyme, la durée minimum d'attaque et les caractéristiques d'emploi du système enzymatique, en fonction de sa provenance. La méthode de dosage de l'amidon par hydrolyse enzymatique a été appliquée avec succès à des amidons purs. Dans les milieux complexes, elle fournit des résultats voisins de ceux obtenus avec les méthodes polarimétriques d'EWERS modifiée (I962) et d'WarLE et Milner (1944) lorsque celles-ci sont applicjuées sur les produits pour lesquels elles ont été mises au point.

L'hydrolyse enzymatique par la gluco-amylase semble donc constituer une solution satisfaisante au problème du dosage de l'amidon dans des milieux complexes. Cependant les gluco-amylases présentant, selon leur provenance, des variations d'activité, il faut définir les conditions d'utilisation de chacune d'elles.

\section{INTRODUCTION}

L'amidon est dosé dans de nombreuses substances à des fins très diverses. Les aliments amylacés constituent la principale source d'énergie pour le monogastrique et représentent environ les deux tiers des aliments concentrés, consommés par les ruminants. Ceci explique l'intérêt du dosage de l'amidon dans les études d'ordre nutritionnel. Par ailleurs, les industries qui utilisent ou transforment l'amidon et

Annales de Biologie animale. - 1965. 
les produits amylacés ont constamment besoin de méthodes de dosage rapides et spécifiques. Celles-ci peuvent avoir en outre un intérêt commercial, puisque la valeur marchande de certains produits dépend directement de leur teneur en amidon (sons, remoulages).

Les méthodes actuellement utilisées diffèrent essentiellement par le mode de dispersion de l'amidon : empesage, action de certains acides (chlorhydrique, perchlorique), action de certains sels (chlorure de calcium, carbonate d'ammonium). A la suite de la dispersion, on peut déterminer la teneur en amidon :

- par lecture directe du pouvoir rotatoire de la solution après défécation (EWERs, IgIo ; EARLE et MIINER, I944);

- par utilisation de l'action saccharifiante des amylases végétales (malt) ou animales (salivaire, pancréatique) (NEven et FouAssin, I962; Ten Bokkei, HuiNINK, I946) et dosage du pouvoir réducteur ;

- par isolement de l'amidon sous forme de son complexe avec 1'iode. On termine le dosage soit en titrant l'iode fixé (DENNy, I922 ; Sullivan, I935) soit en détruisant le complexe amidon-iode et en déterminant les sucres formés après hydrolyse acide (PUCHER, I,EAVENWORTH et VICKERY, I948) ou enzymatique (HASSID, Mac Cready et Rosenfeid, I940). On peut également mesurer le pouvoir rotatoire de la solution obtent1e après une nouvelle dispersion de l'amidon (STEINER et GUTHRIE, I944).

Sur le plan général, aucune de ces méthodes n'est absolument satisfaisante. Celles qui utilisent une dispersion de l'amidon en milieu acide, suivie d'une mesure du pouvoir rotatoire ou d'une précipitation à l'iode, risquent de dégrader trop profondément les grosses molécules, ce qui peut modifier le pouvoir rotatoire ou provoquer la formation de dextrines non précipitables à l'iode. De plus, lorsqu'on procède à une hydrolyse acide de l'amidon dans un milieu complexe, on peut voir apparaître, par dégradation de certains constituants membranaires (substances pectiques, hémicelluloses), des oses ou des oligoholosides réducteurs qui interfèreront au cours du dosage final par les méthodes classiques (pouvoir réducteur, coloration des dérivés furfuriques).

Compte tenu du développement de l'enzymologie et des critères de pureté dont on dispose vis-à-vis des préparations d'enzymes industrielles, nous avons entrepris la mise au point d'une méthode générale de dosage de l'amidon par voie enzymatique, à la fois spécifique, rapide et d'exécution facile. Pour présenter une amélioration par rapport aux précédentes, cette méthode devait utiliser un enzyme ou un mélange d'enzymes dégradant tout l'amidon et lui seul, en produits déterminés, faciles à doser, en un temps suffisamment court et d'une manière assez simple pour permettre des dosages répétés.

Après avoir utilisé des $\alpha$-amylases bactériennes ou fongiques avec lesquelles nous avons constaté une dégradation de l'amidon trop lente et incomplète, nous avons employé une préparation de gluco-amylase. Cet enzyme hydrolyse les liaisons $\alpha(\mathrm{I} \rightarrow 4)$ des chaînes d'anhydroglucose, $\mathrm{I}_{5}$ à 30 fois plus rapidement que les liaisons $\alpha(\mathrm{I} \rightarrow 3)$ et $\alpha(\mathrm{I} \rightarrow 6)$. Il peut donc transformer en glucose, de façon quasi spécifique, l'amidon, le glycogène et les dextrines ou oligoholosides qui en dérivent.

Le présent travail a pour but de préciser les conditions d'emploi de la glucoamylase pour obtenir une transformation quantitative de l'amidon en glucose, soit 
sur de 1'amidon pur, soit dans des milieux complexes. Les résultats obtenus par cette méthode sont comparés à ceux fournis par celles d'EWERs modifiée (I962) et d'EARLE et MILNER (I944).

\section{MATÉRIEL ET MÉTHODES}

\section{Matériel d'étude utilisé}

Préparations enzymatiques.

Les préparations de gluco-amylase utilisées sont extraites soit de Rhizopus delemar (1), soit d'Aspergillus niger. Leur $\mathrm{pH}$ optimum se situe entre 4,0 et 5,0 à une température voisine de $60^{\circ} \mathrm{C}$.

\section{Substrat.}

- amidons : nous avons utilisé 4 amidons préparés industriellement (blé, mais, manioc, pomme de terre) dont la composition est indiquée dans le tableau I ;

\section{TABLEAU I}

Composition chimique des quatre amidons commerciaux utilisés (p. roo de la matière sèche)

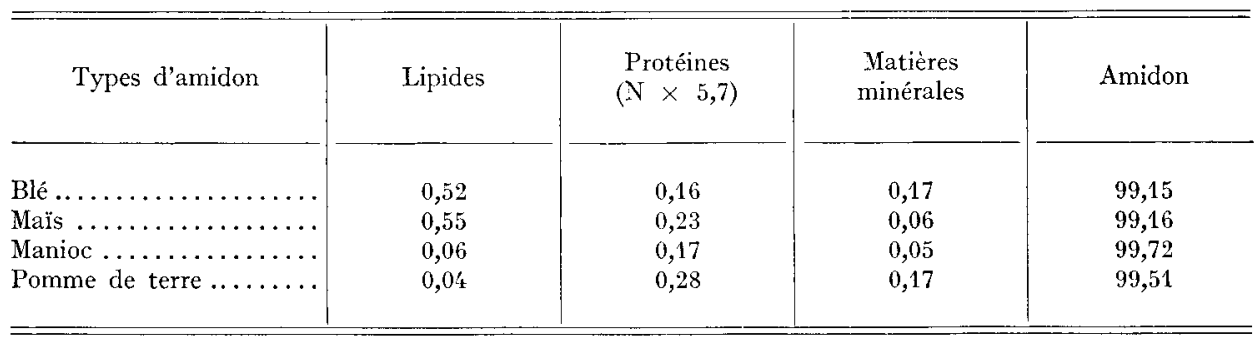

— autres produits étudiés : les autres produits étudiés (céréales, légumineuses, tourteaux, aliments composés) ont été broyés de façon à traverser un tamis à mailles de $\mathrm{I} \mathrm{mm}$.

\section{Méthode proposée}

\section{Principe.}

L'échantillon à analyser subit un traitement hydrothermique qui détruit la structure des grains d'amidon. Il est ensuite mis en présence de gluco-amylase qui transforme quantitativement l'amidon en glucose.

Celui-ci est dosé par une méthode colorimétrique, utilisant un enzyme spécifique, la glucoseoxydase.

\section{Réactifs.}

- préparation de gluco-amylase provenant de Rhizopus delemar ou d'Aspergillus niger, dissoute dans l'eau distillée ;

(1) Ce produit est fabriqué par la société Shim Nihon Chemical Co Ldt 80 Saizuchi Showomachi, Anjo City, Aichi, Japon et commercialisé en Europe par la firme "Equitra ", 37, rue de la Loi, Bruxelles, Belgique. 
- tampon acide acétique, acétate de sodium $2 \mathrm{MI}(\mathrm{pH}=4,6)(\mathrm{r} 20 \mathrm{ml}$ d'acide acétique glacial et I64 $\mathrm{g}$ d'acétate de sodium anhydre par litre);

- merthiolate de sodium (thiosalicylate d'éthyle de sodium et de mercure), dissout dans l'eau distillée ;

- tampon Tris (dissoudre 6r $\mathrm{g}$ de trihydroxyméthylaminométhane dans $85 \mathrm{ml} \mathrm{d} H \mathrm{HCl} 5 \mathrm{~N}$; compléter à I litre avec de l'eau bidistillée) ;

- mélange enzyme, tampon, chromogène (1) dilué dans une solution de tampon Tris;

- solution d'acide $5 \mathrm{~N}$ en eau bidistillée (acide chlorhydrique ou sulfurique);

- solution étalon de glucose anhydre en eau bidistillée.

\section{Mode opératoire (cas d'un amidon préparé industriellement).}

a) préparation de l'échantillon et dispersion de l'anidon.

L'échantillon ayant été broyé en particules de diamètre inférieur à I mm, en peser, à o, I mg près, environ $500 \mathrm{mg}$ dans un erlenmeyer de $100 \mathrm{ml}$ préalablement taré. Ajouter progressivement 20 à $25 \mathrm{ml}$ d'eau distillée pour bien disperser le produit. Porter à ébullition pendant 3 minutes, puis autoclaver à $130^{\circ} \mathrm{C}$ pendant une heure (pression de $\mathrm{I}, 8 \mathrm{~kg} / \mathrm{cm}^{2}$ ). Neutraliser éventuellement la suspension, avant la dispersion de l'amidon, si le $\mathrm{pH}$ est inférieur à $6, \mathrm{o}$.

\section{b) Dégradation de l'amidon par la gluco-amylase (Rhizopus delemar).}

Après refroidissement, ajouter à la dispersion 2,5 $\mathrm{ml}$ d'une solution de tampon acétate pour que la concentration finale du milieu soit de $\mathrm{M} / \mathrm{ro}$ en tampon. Ajouter en outre du merthiolate de sodium à raison de $\mathrm{I} / \mathrm{10}$ 000, pour éviter le développement des micro-organismes. Par pesée, amener à un volume de $45 \mathrm{ml}$ avec de l'eau distillée. P'lacer alors l'erlenmeyer dans un bain-marie agitateur, à la température de $55^{\circ} \mathrm{C}$ et introduire, au temps zéro, $5 \mathrm{ml}$ de la solution d'enzyme à $\mathrm{r}, 5 \mathrm{p}$. 100 . Laisser agir pendant 5 heures, puis filtrer la suspension sur un filtre plissé, dans une fiole jaugée de $250 \mathrm{ml}$. Laver quantitativement le filtre et le résidu à l'eau distillée. Compléter à $250 \mathrm{ml}$, puis déterminer la teneur en glucose de la solution.

\section{c) Dosage du glucose.}

La teneur en glucose du filtrat est déterminée selon la méthode colorimétrique de HuGGETT (I957) modifiée :

A I $\mathrm{ml}$ de solution de glucose à doser, ajouter $5 \mathrm{ml}$ du mélange enzyme-tampon-chromogène. La réaction se développe à l'obscurité, à température voisine de $20^{\circ} \mathrm{C}$ et pendant exactement 45 minutes. Bloquer ensuite la réaction :

- soit par $0,25 \mathrm{ml}$ d'HCl $5 \mathrm{~N}$ et mesurer à $400 \mathrm{~m} \mu$ l'intensité de la coloration jaune obtenue ;

- soit par $5 \mathrm{ml} \mathrm{de} \mathrm{H}_{2} \mathrm{SO}_{4} 5 \mathrm{~N}$ et mesurer à $525 \mathrm{~m} \mu$ l'intensité de la coloration rose.

Dans ces conditions, la coloration est stable pendant plusicurs heures (Guidottr, Colombo et FOA, I96I ; FLeming et PEgler, i963). La meilleure zone de concentration pour ce dosage se situe entre 20 et $60 \gamma$ pour $\mathrm{I} \mathrm{ml}$ de prise d'essai. Les solutions de glucose pour la courbe-étalon et pour le dosage proprement dit doivent être préparées avec de l'eau bidistillée. Il est nécessaire de refaire un étalonnage pour chaque série de dosages.

\section{Expression des résultats.}

\section{Soient :}

M, la masse, en gramme, de la prise d'essai ;

$\mathrm{V}$, le volume, en millilitres, du prélèvement dans les $250 \mathrm{ml}$;

$\mathrm{V}^{\prime}$, le volume, en millilitres, après dilution telle que I ml contienne entre zo et $60 \gamma$ de glucose ; $m$, la masse, en microgrammes de glucose, relevée sur la courbe d'étalonnage.

Le pourcentage d'amidon dans l'échantillon est égal à :

$$
0,9 \cdot \frac{m}{\mathrm{IO}^{6}} \cdot \frac{\mathrm{V}^{\prime}}{\mathrm{I}} \cdot \frac{250}{\mathrm{~V}} \cdot \frac{\mathrm{IOO}}{\mathrm{M}}=\frac{0,225 \times m \times \mathrm{V}^{\prime}}{\mathrm{V} \cdot \mathrm{M}}
$$

(1) Ce málange est trouvé dans le commerce sous les appellations :

- Réactif Boehringer, Biochemica "Boehringer" Mannheim. Boehringer und Soehne GmbH Mannheim.

- Réactif Biotrol : Bio Dubernard, rue du lioin, Paris-4 $4^{\mathrm{e}}$. 


\section{RÉSULTATS}

\section{Justification des conditions opératoires}

Détermination des conditions optimales d'hydrolyse.

Le tableau 2 indique qu'en se plaçant dans les conditions optimales de température et de $\mathrm{pH}$, il faut effectuer, avant l'hydrolyse, un empesage de 3 minutes, puis un autoclavage d'une heure à $130^{\circ} \mathrm{C}$, pour obtenir une dispersion satisfaisante de l'amidon. I'empesage ou l'autoclavage seul est insuffisant.

\section{TABLEAU 2}

Influence du traitement hydrothermique sur la quantité d'amidon degradé après une hydrolyse enzymatique de 5 heures

(p. roo de la matière sèche)

\begin{tabular}{|c|c|c|c|c|c|c|c|}
\hline \multirow{2}{*}{$\begin{array}{l}\text { Traitement } \rightarrow \\
\text { Amidon } \\
\quad \downarrow\end{array}$} & \multirow{2}{*}{ Natif } & \multirow{2}{*}{$\begin{array}{l}\text { Limpesage } \\
\text { de } 6 \mathrm{mn}\end{array}$} & \multicolumn{2}{|c|}{$\begin{array}{c}\text { Autoclavage } \\
\text { à } 130^{\circ} \mathrm{C}\end{array}$} & \multicolumn{3}{|c|}{$\begin{array}{c}\text { Empesage de } 3 \mathrm{mn} \\
+ \text { autoclavage à } 130^{\circ} \mathrm{C}\end{array}$} \\
\hline & & & $30 \mathrm{mn}$ & $60 \mathrm{mn}$ & $30 \mathrm{mn}$ & $60 \mathrm{mn}$ & $90 \mathrm{mn}$ \\
\hline Maïs ......... & 21,2 & 95,0 & 97,6 & 97,2 & 97,9 & 100 & \\
\hline Pomme de terre & 8,2 & 83,3 & 99,5 & 99,3 & $99,{ }^{\prime} k$ & 100 & 100 \\
\hline
\end{tabular}

La concentration de la préparation enzymatique, par rapport au substrat peut varier avec son origine ou son degré de purification. Cette concentration est voisine de ro $\mathrm{p}$. Ioo pour la préparation enzymatique de gluco-amylase d'Aspergillus niger et de $5_{5}$ p. roo pour celle provenant de Rhizopus delemar (tabl. 3). Si l'on définit

\section{TABLEAU 3}

Influence de la concentration en enzyme sur la quantité d'amidon dégradé après une hydrolyse de 5 heures

(p. Ioo de la matière sèche)

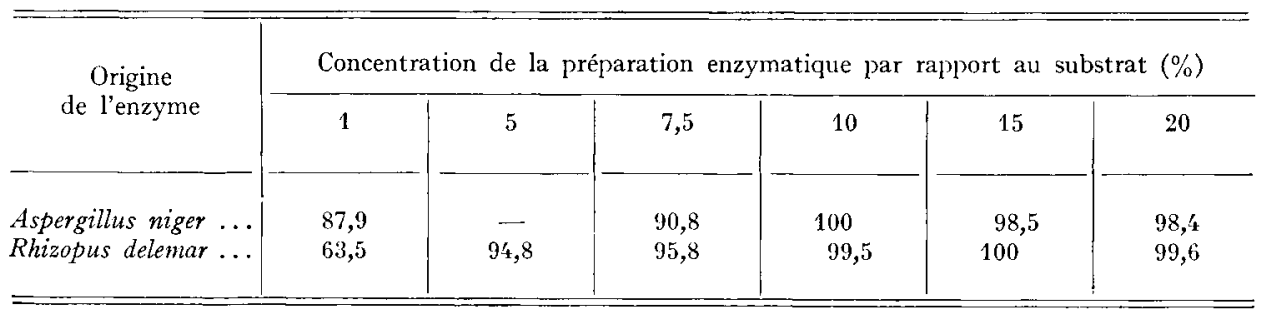


les modalités d'hydrolyse pour chaque gluco-amylase utilisée, on obtient des résultats analogues quelle que soit l'origine de l'enzyme (tabl. 4).

L'étude de la cinétique de l'action de la gluco-amylase sur des amidons purs (tab1. 5) permet de penser qu'au bout de deux heures d'attaque, la dégradation de l'amidon est totale. Les différences observées entre les diverses durées d'hydrolyse ne sont pas significatives, mais par mesure de sécurité, le temps d'hydrolyse a été fixé à 5 heures.

\section{TABLEAU 4}

Comparaison de l'action de deux gluco-amylases d'origine différente sur divers amidons

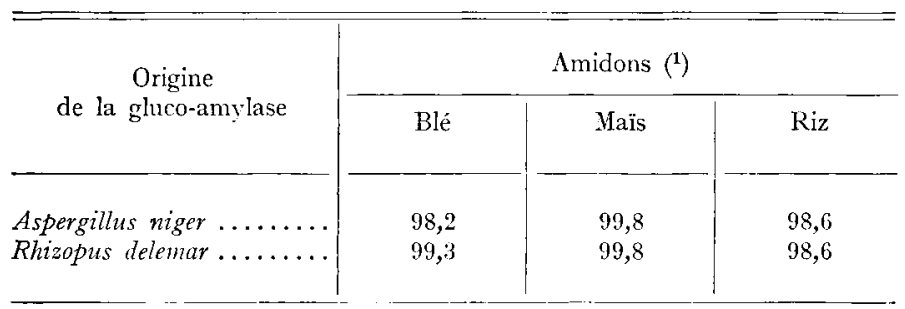

(1) Les résultats sont exprimés en \% de la matière sèche de l'amidon brut.

TABLEAU 5

Cinétique de l'hydrolyse des amidons purs par la gluco-amylase (p. roo de la matière sèche)

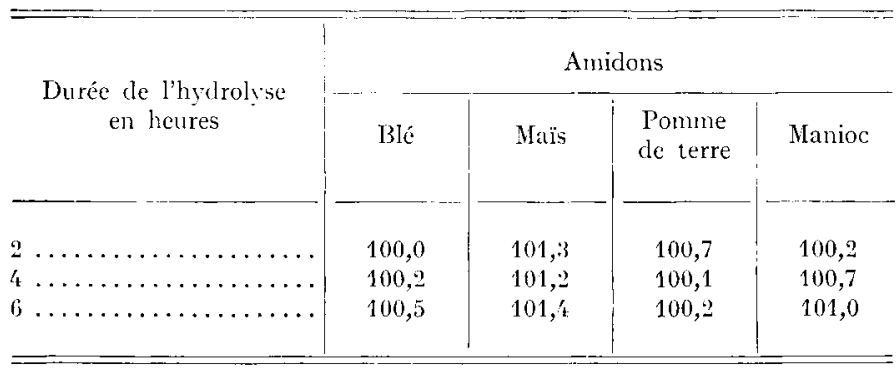

Vérification de la spécificité de l'enzyme.

Différents auteurs (PAzUR et ANDo, I959) ont constaté que la gluco-amylase utilisée dans certaines conditions de concentration, sur des oligosides, présente une activité de trans-glucosidase. Par contre, dans les conditions précédemment définies et avec les préparations enzymatiques utilisées, l'examen chromatographique des produits d'hydrolyse de l'amidon (fig. I) permet d'affirmer que s'il se forme des produits de réversion, ils représentent moins de I p. Ioo du total. 
Par ailleurs, pour étudier la spécificité de la gluco-amylase vis-à-vis de substances autres que les composants glucidiques de l'amidon, nous avons vérifié qu'en faisant agir la préparation enzymatique dans les conditions précédemment indiquées, elle était capable d'hydrolyser les dextranes, mais elle ne fournissait avec la cellulose, l'inuline, le mélibiose, le raffinose, le lactose, aucun produit dosable par la méthode à la glucose-oxydase. Cependant, la spécificité de la préparation enzymatique

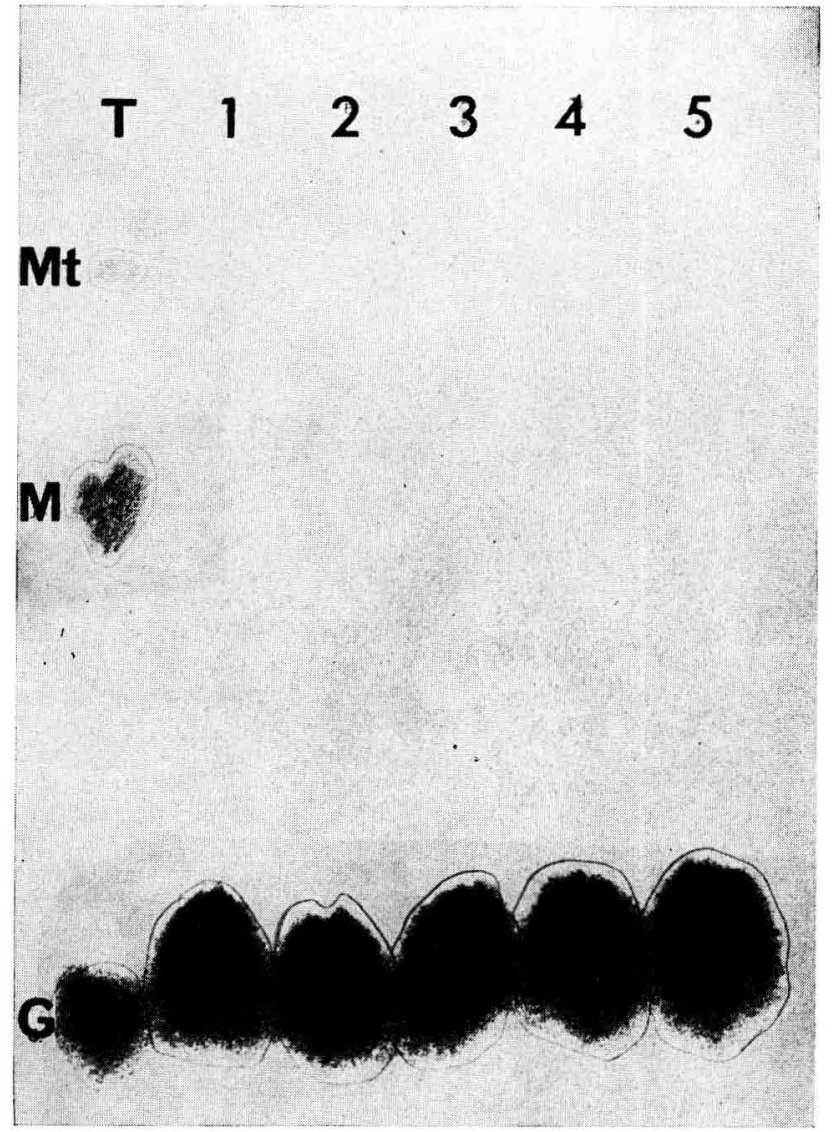

FIG. I. - Chromalogramme des attaques à la gluco-amylase de différents amidons

dépôt de 2 ooơ;

solvant : butanol, acide acétique, eau 4-I-5, pendant $72 \mathrm{~h}$.
I. blé
G: glucose
2. maîs
$\mathrm{M}:$ maltose
3. pomme de terre
Mt : maltotriose
4. manioc
5. pois ridé

peut varier avec l'origine de la gluco-amylase ; c'est ainsi que si le saccharose n'est pas hydrolysé par la gluco-amylase issue de Rhizopus delemar, il l'est par celle provenant d'Aspergillus niger. 


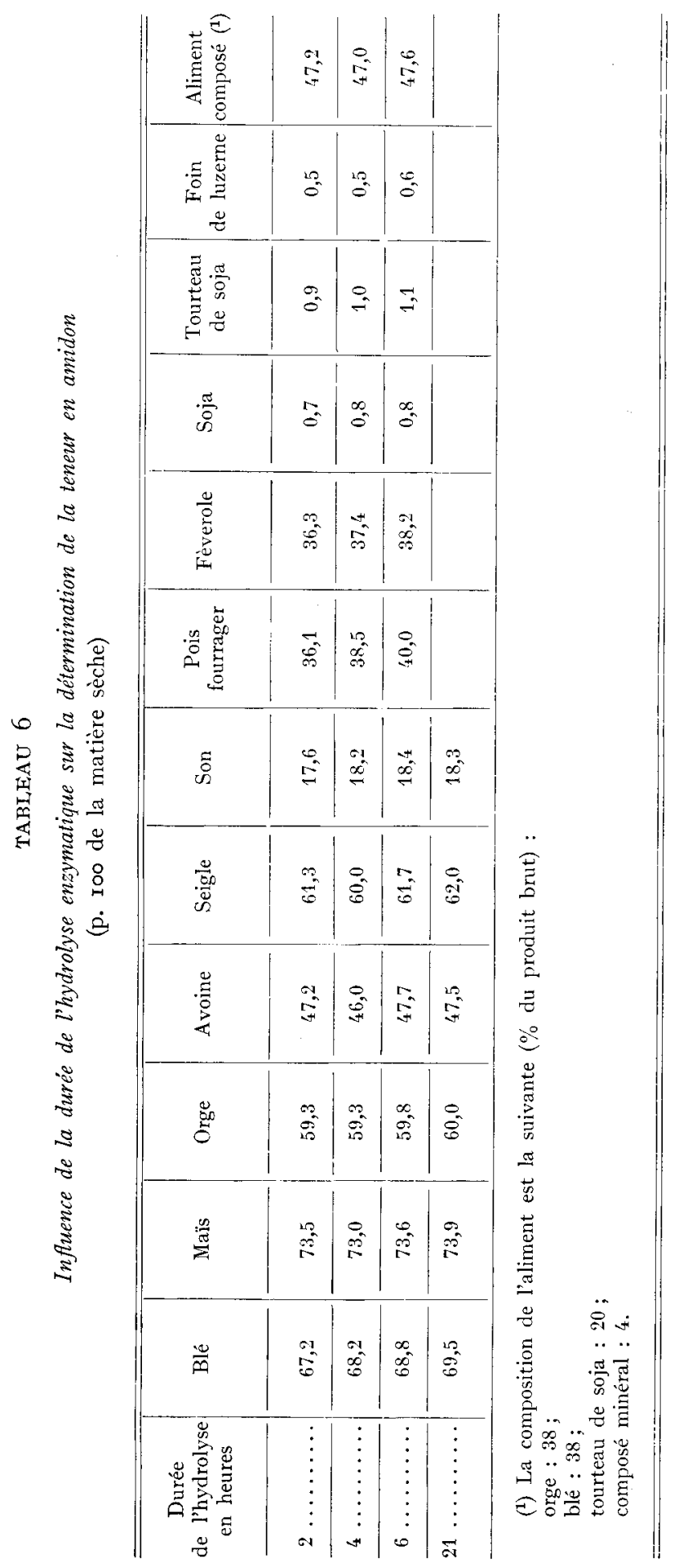




\section{Application aux milieux complexes}

Durée optimum d'hydrolyse.

A la suite des résultats obtenus sur des amidons purs, nous avons étudié la cinétique de l'action de la gluco-amylase sur les catégories d'aliments suivants : céréales, légumineuses, tourteaux, foin, aliments composés (tab1. 6). Dans la plupart des cas, il semble que la dégradation soit plus lente que celle observée avec les amidons purs et qu'il faille poursuivre l'hydrolyse au moins pendant 4 heures. Mais il paraît superflu de continuer au-delà puisque les valeurs observées au bout de 6 heures et de 2 I heures ne sont pas significativement différentes de celles obtenues au bout de 4 heures. C'est pourquoi, comme dans le cas des amidons purs, le temps d'hydrolyse optimum a été fixé à 5 heures.

Quantité de matière sèche dissoute pendant l'hydrolyse.

L,es résidus insolubles de 1'hydrolyse enzymatique de divers amidons (blé, riz, pomme de terre), des céréales et des légumineuses étudiées ont été séchés, puis pesés (tabl. 7) afin d'étudier si la quantité de matière sèche dissoute, déduction faite de celle extraite par l'eau sur un témoin, fournit une estimation satisfaisante de l'amidon dégradé. Les résultats sont comparables à ceux obtenus par dosage du glucose dans les hydrolysats, pour les céréales qui sont des produits riches en amidon et pauvres en constituants solubles dans le milieu tampon utilisé. Dans les autres cas (légumineuses) il semble que la dissolution de certains constituants (protéines) soit trop importante au cours du dosage pour que la quantité de matière sèche dissoute puisse être considérée comme une indication valable de la teneur en amidon du produit étudié.

\section{TABLEAU 7}

Comparaison des résultats obtenus par dosage du glucose formé et par pesée du résidu insoluble après hydrolyse enzymatique

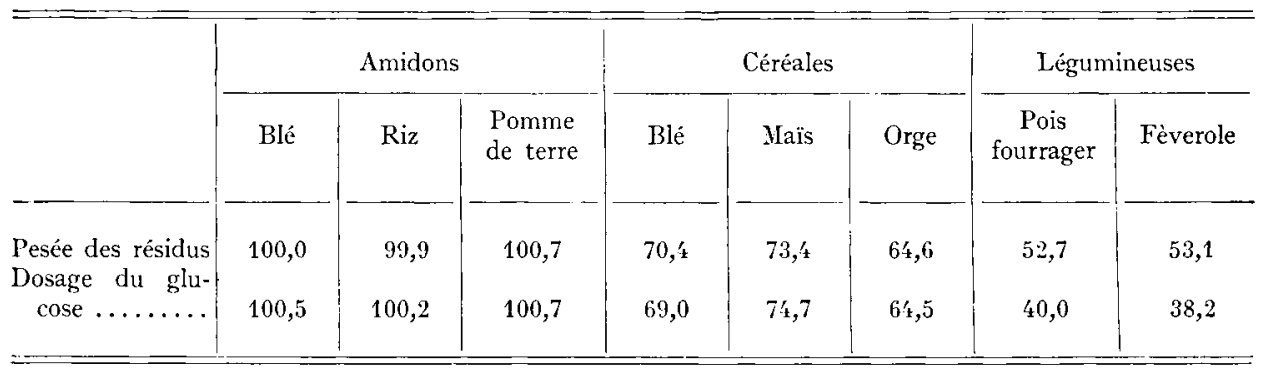

\section{Comparaison de la méthode enzymatique à deux méthodes polarimétriques.}

Nous avons comparé la méthode enzymatique aux méthodes polarimétriques d'EARLE et MILNER (I944) et d'EWERs modifiée (I962) (tabl. 8 et 9). Les résultats fournis par la méthode enzymatique, en particulier pour l'amidon, sont systématiquement plus élevés que ceux obtenus par la méthode d'EARIE et MILNER ; jls 


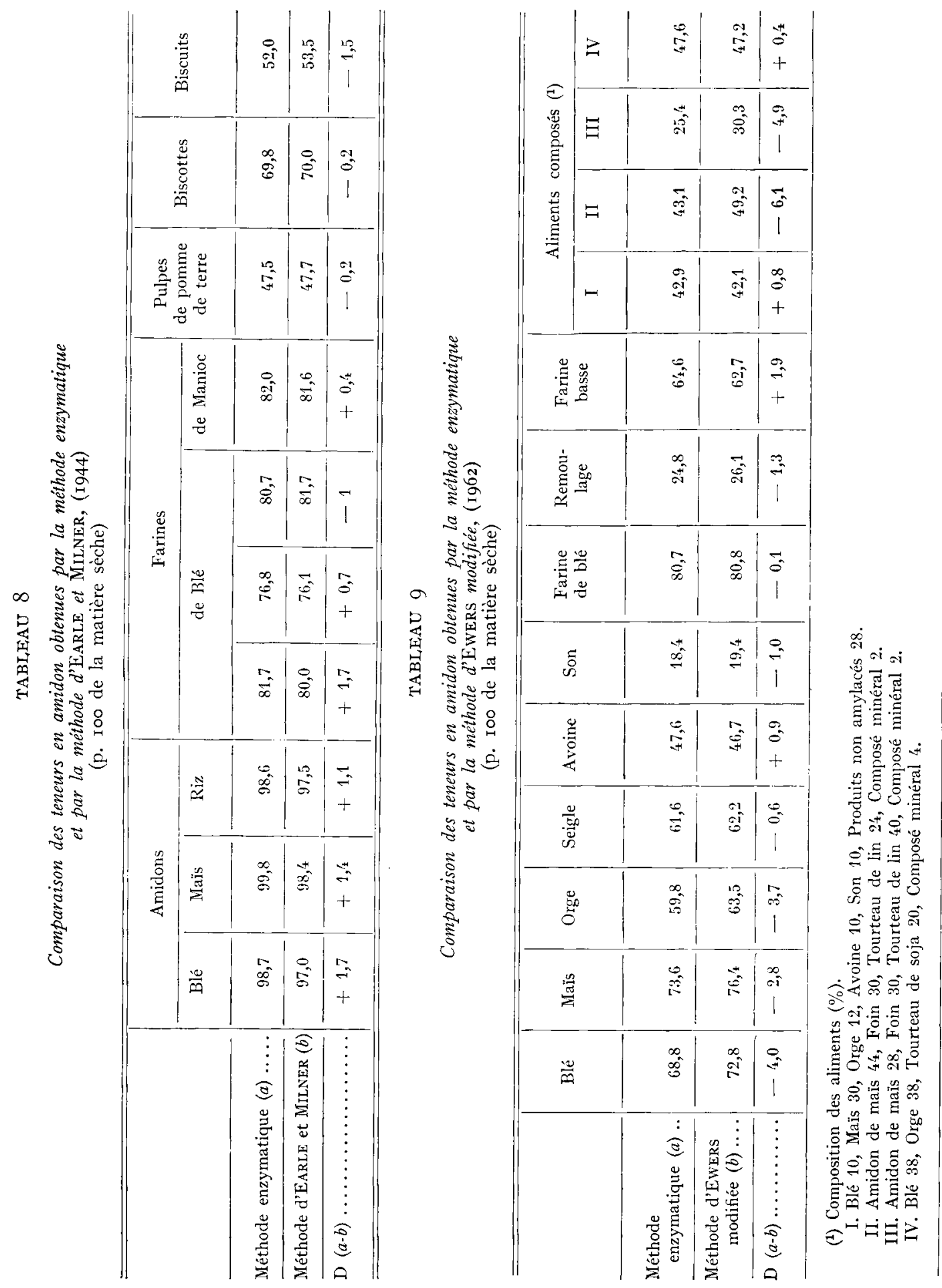


correspondent mieux aux quantités prévisibles, compte tenu des impuretés dosables dans les amidons commerciaux. La méthode d'EwERs, même améliorée par 1'extraction des oses et oligoholosides solubles dans l'alcool à 40 p. Ioo, fournit des résultats sensiblement plus élevés que la méthode enzymatique, surtout dans les produits complexes.

\section{DISCUSSION}

Déterminer spécifiquement l'amidon quelles que soient les autres substances présentes et le taux d'amidon dans le produit, tel doit être le but de toute méthode de dosage de lamidon dans un milieu complexe. Avec la méthode que nous proposons, ces conditions semblent être réalisées, sans pour autant que la durée de la manipulation en soit augmentée.

L'autoclavage qui est effectué pour l'ensemble des substrats, à $\mathrm{I} 30^{\circ} \mathrm{C}$ pendant une heure, doit être précédé d'un empesage partiel homogénéisant 1'amidon dans son milieu de dispersion. Pour certains produits (mais riche en amylose, poids ridés) dont les grains d'amidon peuvent résister à un tel traitement, il sera préférable d'opérer à une température plus élevée, pendant un temps plus long.

La durée d'hydrolyse varie avec les différents produits. Si la dégradation est totale avec les amidons commerciaux, il semble que pour l'ensemble des autres prođuits étudiés, elle ne soit complète qu'au bout de 4 heures. Ces durées d'hydrolyse plus ou moins longues peuvent s'expliquer par la présence d'agrégats, et par le fait que les protéines ou les autres constituants du produit, peuvent gêner le contact enzyme-substrat. En conséquence, compte tenu des résultats précédents et de la précision recherchée, on peut fixer la durée optimum d'hydrolyse à 5 heures.

Selon son origine, nous utilisons la gluco-amylase à une concentration de co ou I5 p. Ioo par rapport au substrat, après avoir vérifié qu'en deçà de ce chiffre la durée d'hydrolyse est plus longue. Cette concentration étant suffisante dans le cas où le substrat est un amidon pur, elle le sera a fortiori pour tous les autres produits moins riches en amidon. Cependant il ne faut pas dépasser les normes indiquées pour l'enzyme utilisé. En effet, ainsi que nous l'avons observé, certaines gluco-amylases peuvent présenter, selon leur condition d'emploi, une activité de trans-glucosidase, aboutissant à la formation de polymères du glucose contenant des liaisons $\alpha(\mathrm{I} \rightarrow 6)$ (isomaltose, panose) à partir principalement du maltose. Actuellement, les différentes préparations industrielles de gluco-amylase ont chacune leurs caractéristiques propres d'utilisation. Lorsqu'on emploiera une nouvelle gluco-amylase, il faudra, d'une part, déterminer sur un amidon pur la concentration optimum à laquelle on doit utiliser cet enzyme, d'autre part, s'assurer qu'à cette concentration l'activité de la trans-glucosidase est nulle. Ultérieurement, dans un but de standardisation, il conviendra de choisir la préparation enzymatique la plus appropriée (origine, activité, spécificité, stabilité).

La spécificité de la gluco-amylase vis-à-vis des différents glucides renfermant des liaisons glucosidiques a été étudiée en détail par PAZUR et KLEPPE (I962). Ces auteurs ont montré que si la gluco-amylase hydrolyse les liaisons $\alpha(I \rightarrow 4), \alpha(I \rightarrow 6)$ et $\alpha(\mathrm{I} \rightarrow 3)$ de nombreux oligoholosides, les vitesses de dégradation sont très différentes suivant le mode de liaison (tabl. Io). En outre ABDUlLAH et al. (I963) ont 
montré que si la vitesse d'hydrolyse des liaisons $\alpha(I \rightarrow 4)$ et $\alpha(I \rightarrow 6)$ augmente avec le poids moléculaire du substrat, il n'en est pas ainsi pour les liaisons $\alpha(\mathrm{I} \rightarrow 3)$ dont la vitesse de dégradation reste toujours faible vis-à-vis des deux autres. Dans cas conditions, la présence d'oligoholosides autres que l'amidon et susceptible d'être attaqués par la gluco-amylase ne constitue pas une source d'erreur importante pour la méthode proposée puisque priorité semble être donnée à l'hydrolyse des liaisons $\alpha(\mathrm{I} \rightarrow 4)$ et $\alpha(\mathrm{I} \rightarrow 6)$ des macromolécules d'anhydroglucose. Cependant, dans certains cas, on pourra avoir des résultats en excès, en présence de dextranes, dans lesquels les molécules de glucose sont reliées par des chaînes $\alpha(\mathrm{I} \rightarrow 3)$ et $\alpha(\mathrm{I} \rightarrow 6)$.

\section{TABLEAU IO}

$V$ itesses relatives d'hydrolyse des $\alpha-D$ glucosides par la gluco-amylase purifiée (d'après Pazur J. H. et Kleppe K., I962)

\begin{tabular}{|c|c|c|c|c|}
\hline$\alpha-\mathrm{D}$ glucoside & Structure & $\begin{array}{r}\text { D.gl } \\
\text { lib } \\
\text { (mg/uni }\end{array}$ & $\begin{array}{l}\text { lucose } \\
\text { béré } \\
\text { ité/heure) }\end{array}$ & $\begin{array}{c}\text { Vitesse relative } \\
\text { d'hydrolyse }\end{array}$ \\
\hline Maltose & $\begin{array}{c}4 \alpha-\mathrm{D} \text { glucopyranosyl } \\
\text { D glucose }\end{array}$ & $2,3>$ & $\times 10^{-1}$ & 100 \\
\hline Acide maltobionique & $\begin{array}{l}4 \alpha-\mathrm{D} \text { glucopyranosyl } \\
\mathrm{D} \text { acide gluconique }\end{array}$ & 0,1 & $\times 10-1$ & $3 \prime$ \\
\hline Nigerose & $\begin{array}{c}3 \alpha-\mathrm{D} \text { glucopyranossl } \\
\mathrm{D} \text { glucose }\end{array}$ & 1,5 & $\times 10-^{2}$ & 6,6 \\
\hline Isomaltose & $\begin{array}{c}6 \alpha-\mathrm{D} \text { glucopyranosyl } \\
\text { D glucose }\end{array}$ & 0,83 & $\times 10-2$ & 3,6 \\
\hline Arabinosyl glucoside & $\begin{array}{c}3 \alpha-\mathrm{D} \text { glucopyranosyl } \\
\mathrm{D} \text { arabinose }\end{array}$ & 0,19 & $\times 10-^{2}$ & 1,66 \\
\hline Maltulose & $\begin{array}{c}\text { i } \alpha-D \text { glucopyranosyl } \\
D \text { fructose }\end{array}$ & 0,16 & $\times 10-^{2}$ & 1,39 \\
\hline Saccharose & $\begin{array}{l}\alpha-\mathrm{D} \text { glucopyranosyl } \\
\beta-\mathrm{D} \text { fructofuranoside }\end{array}$ & 0,16 & $\times 10^{2}$ & 1,39 \\
\hline Phényl glucoside & Phényl $\alpha-D$ glucoside & 0,10 & $\times \quad 10-^{2}$ & 0,87 \\
\hline Glycérol glucoside & $\begin{array}{c}1 \alpha-\dot{D} \text { glucopyranosyl } \\
D \text { glycérol }\end{array}$ & 0,10 & $\times 10-2$ & 0,87 \\
\hline Furanose & $\begin{array}{c}3 \alpha-\mathrm{D} \text { crlucopyranosyl } \\
\mathrm{D} \text { fructose }\end{array}$ & 0,77 & $\times 10-^{3}$ & 0,67 \\
\hline Tréhalose & $\begin{array}{l}\alpha-D \text { glucopyranosyl } \\
\alpha-D \text { glucopyranoside }\end{array}$ & 0,17 & $\times 10-^{3}$ & 0,074 \\
\hline
\end{tabular}

La méthode que nous proposons ne permet pas, dans un produit complexe de faire la distinction entre le glycogène et l'amidon. Dans les milieux susceptibles de contenir du glucose, des oligosides dérivés du glucose, et de l'amidon, il conviendra de modifier le mode opératoire selon les fractions que l'on désire doser :

- si l'on veut déterminer l'amidon, il faudra procéder à une extraction aqueuse ou alcoolique (alcool $40 \mathrm{p}$. IOo) pour éliminer dextrines et sucres, tout en évitant les actions amylasiques éventuelles pendant l'opération. On effectuera le dosage de l'amidon sur le résidu ;

- si l'on désire déterminer l'ensemble amidon et dextrines, une extraction alcoolique (alcool 80 p. I0o) éliminera le glucose et les autres oligoholosides. 
Les méthodes polarimétriques d'EWERs et d'EARLE et MILnER ne sont applicables en général qu'à des produits riches en amidon (céréales) et ne contenant pas de fortes quantités de matières cellulosiques, de protéines ou de lipides. Avec les produits pour lesquels elle a été mise au point, la méthode d'EARLE et MiLNER donne des résultats qui ne sont pas significativement différents de ceux obtenus par hydrolyse enzymatique. Il semble, par conséquent, que les deux méthodes soient équivalentes pour certaines céréales (maïs, blé, et leurs dérivés riches en amidon), la méthode enzymatique étant toutefois plus facile à utiliser et son domaine d'application étant beaucoup plus vaste.

La méthode d'EWERs donne dans certains cas, des valeurs sensiblement supérieures à celles obtenues avec la méthode enzymatique. Il conviendrait de préciser les causes de cette différence. Paut-être faut-il voir là, un effet de l'hydrolyse chlorhydrique susceptible de transformer des constituants autres que l'amidon en produits modifiant le pouvoir rotatoire de la solution.

En conclusion, l'application de la gluco-amylase à l'hydrolyse enzymatique de l'amidon, suivie du dosage du glucose formé par action de la glucose-oxydase, constitue une méthode de dosage spécifique, rapide, d'utilisation facile et applicable à la majorité, sinon à la totalité des produits renfermant de l'amidon.

Reçu pour publication en septembre 1965.

\section{SUMMARY}

\section{ESTIMATION OF STARCII IN COMPLEX MEDIA}

There are many methods of estimating starch but none of them is at the same time specific and rapid. In an attempt to find a method with both these qualities recourse was made to estimation with enzymes. The enzyme used was a glucoamylase of fungal origin which under defined conditions of temperature, $p H$ and concentration quantitatively degrades starch into glucose.

The product to be studied is first treated with hot water then autoclaved at $130^{\circ} \mathrm{C}$ for one hour, to destroy the structure of the starch grain and make it more easily accessible to the enzyme. Hydrolysis is at $55^{\circ} \mathrm{C}$ and $p H 4.8$ for 5 hours. (ilucose is then estimated in the hydrolysate by a modification of the method of HugGETT (19.57).

It was verified that the glucoamylase degrades starch to glucose only and that no reversion product was formed, which showed that another enzyme, transglucosidase, was not present. The time necessary for complete hydrolysis first of pure starches and then of different categories of starchy feeds (tables 5 and 6 ) were estimated. The method described was compared with the modified polarimetric method of EARLE and MILNER (I944) and that of EWERS modified (I962) (tables 8 and 9). The specificity of glucoamylase means that it may be applied to all vegetable products containing starch, cereals, legumes, roots, etc. whatever their starch content. The polarimetric methods of EWERs (I962) and of FARLE and MiLNER (I944) give similar results but can only be applied to particular classes of products, cereals and flours.

Enzymic hydrolysis by glucoamylase seems to be a satisfactory solution to the problem of estimating starch in complex media. However, since activity of glucoamylases may differ according to their source, it is necessary, for a given enzyme, to define the conditions in which it is used by trials with pure starch.

\section{RÉFÉRENCES BIBLIOGRAPHIQUES}

Abdullah M., Fleming I. D., Taylor P. M., Whelan W. J., I963. Substrate specificity of the amyloglucosidase of Aspergillus niger. Bioch. J., 89, $35 \mathrm{P}$.

Denny F. E., 1922, in Radley J. A., 1953. Starch and its derivatives, vol II, 359, 3 d, ed. London, Chapman and Hall Ltd. $465 \mathrm{p}$. 
EARLe F. R., Milner R. T., I944. Improvements in the determination of starch in corn and wheat. Cereal Chem., 21. $567-575$.

Ewers E., 1910, in RADLey J. A., 1953. Starch and its derivatives, vol. II, 362, 3l, ed. London Chapman and Hall Ltd., 465 p.

Ewers E., I 962 . Communication du Comité d'Experts C. E. E.

Fleming I. D., Pegler H. F., ig63. The determination of glucose in the presence of maltose and isomaltose by a stable, specific enzymic reagent. The Analyst, 88, (1053), 967.

Guidotti G., Colombo J. P., Fon P. P., ig61. Enzymatic determination of glucose : stabilisation of color developed by oxidation of o-dianisidine. Analyt. Chem., 33, I5I-153.

Hassid W. Z., McCready R. M., Rosenfeld R. S., I940. Determination of starch in plants. Ind. Eng. Chem. Anal. Ed., 12, $142-144$.

Huggett A.S. G., Nixon D. A., 1957. Enzymic determination of blood glucose. Bioch. J., 66, I 2 P.

Neven M., Fouassin A., ig62. Méthode de dosage de l'amidon dans les matières fécales. Nutr. Diél. 4, $24 \mathrm{I}-250$.

Pazur J.H., Ando T., i959. The action of an amyloglucosidase of Aspergillus niger on starch and maltooligosaccharides. J. Biol. Chem., 234, I 966-70.

Pazur J. II., Klepre K., I962. The hydrolysis of $\alpha-\mathrm{D}$ glucosides by amyloglucosidase from Aspergillus niger. J. Biol. Chem., 23\%, 1002-1006.

Pucher E. W., Leavenworti C. S., Vickery H. B., r948. Ditermination of starch in plant tissues. Analyt. Chem., 20, 850 .

Steiner E. T., Guthrie I. D., 1944, in PaEch M. V., Tracey, I955. Modern methods of plant analysis. I56, ed. by W. Ruhland, vol. VI, Berlin Springer, $626 \mathrm{p}$.

Sullivan J. T., i 935 in Rabley J. A., i953. Starch and its derivalives, vol. II 359, 3 d ed. London, Chapman and Hall Ltd. $465 \mathrm{P}$.

TEN Bokkel Huinink H., 1946. Over de Vertering van rauw Zetmeel. Thesis J. Hoeijenbos M. V., Utrecht, r6o p. 